\title{
Dynamic Monte Carlo study of isolated-gate InAs/AISb HEMTs
}

\author{
H. Rodilla ${ }^{1}$, T. González ${ }^{1}$, G. Moschetti ${ }^{2}$, J. Grahn² ${ }^{2}$ and J. Mateos ${ }^{1}$ \\ ${ }^{1}$ Departamento de Física Aplicada, Universidad de Salamanca, Plaza de la Merced s/n, \\ 37008 Salamanca, Spain \\ ${ }^{2}$ Microwave Electronics Laboratory, Dept. of Microtechnology and Nanoscience, \\ Chalmers University of technology, Göteborg, Sweden
}

E-mail: helenarsc@usal.es

Abstract - In this work, by means of Monte Carlo simulations, the static and dynamic behavior of isolated-gate InAs/AlSb high electron mobility transistors (Sb-HEMTs) has been studied and compared with experimental results. The influence of the existence of a native oxide under the gate, the value of the surface charges in the gate recess and the possible variation of electron sheet carrier density, $n_{s}$, has been studied. A decrease in the gate-source capacitance, transconductance and intrinsic cut-off frequency is observed because of the presence of the native oxide, while changes in the value of the surface charges in the recess only introduce a threshold voltage shift. The increase of $n_{s}$ shifts the maximum of the transconductance and intrinsic cut-off frequency to higher values of drain current and improves the agreement with the experimental results. 


\section{INTRODUCTION}

The search for improved cut-off frequencies in high electron mobility transistors (HEMTs) pursued by increasing the In content in the channel of "traditional" InGaAs/InAlAs HEMTs suggests the relevance of a new generation of HEMTs based on the InAs/AlSb heterojunction (Sb-HEMTs) [1-4]. The very high mobility of the channel material (InAs), along with the strong confinement of the electrons in the channel due to the large offset in the conduction band $\left(\Delta E_{c}=1.35 \mathrm{eV}\right)$ of the InAs/AlSb heterostructure, allows the Sb-HEMT to achieve the high-frequency, low-noise and low-power requirements demanded for radio-astronomy receivers, passive imaging and satellite communications $[5,6]$.

One of the most important challenges in the development of Sb-HEMTs is to achieve a decrease of the large gate leakage current of these devices. For this sake, a possible approach is to avoid the oxide removal step after the recess etching in the fabrication process, favouring the appearance of a native oxide under the gate [7]. The theoretical study of the macroscopic consequences of introducing this native oxide, and also the physical understanding of the internal microscopic behavior of the device, are essential tasks in the development and optimization of Sb-HEMTs.

Due to the very high mobility of the InAs channel, electron transport can become ballistic or at least quasibalistic, thus making Monte Carlo (MC) simulations the most accurate modelling technique as compared with other approaches like drift-diffusion or hydrodynamic methods. Moreover, MC simulations provide not only the static characteristics and relevant physical information inside the devices, but also their dynamic and noise behavior.

In this work we present the results of MC simulations able to correctly reproduce 
experimental measurements, at low $V_{d s}$ before the onset of impact ionization, of the static behavior and small signal equivalent circuit of isolated-gate Sb-HEMTs. The influence of the native oxide and the associated surface charges in the gate recess, and the variation of $n_{s}$ have been studied, thus providing valuable information for the optimization of the devices.

\section{DEVICE DESCRIPTION AND SIMULATION MODEL}

The active layer for the fabricated isolated-gate Sb-HEMT [6,7] is composed by an AlSb buffer of $800 \mathrm{~nm}$ followed by an InAs channel of $15 \mathrm{~nm}$ and a $15 \mathrm{~nm}$ thick AlSb barrier, a $4 \mathrm{~nm}$ protection layer of In $0.5 \mathrm{Al}_{0.5 \mathrm{As}}$, and a $5 \mathrm{~nm}$ highly Si doped InAs cap $\left(N_{D}=5 \times 10^{18} \mathrm{~cm}^{-3}\right)$ on top. Electrons are introduced by a $\delta$-doping of $\delta=4.5 \times 10^{12} \mathrm{~cm}^{-2}$, $5 \mathrm{~nm}$ far from the channel. To fabricate the $225 \mathrm{~nm}$-gate Sb-HEMTs (2x50 $\mu \mathrm{m}$ wide), the mesa isolation was performed by a chlorine-based dry etching method, then $\mathrm{Pd} / \mathrm{Pt} / \mathrm{Au}$ ohmic contacts were evaporated and annealed, and the T-shaped gate was formed by a tri-layer resist stack defined with electron beam lithography. The recess etching, using citric acid, stops at $9 \mathrm{~nm}$ far from the surface (the top of the AlSb Schottky layer). The typical oxide removal step after the recess etch step has not been performed for these devices, thus favouring the appearance of a native oxide under the gate that decreases the large leakage current of InAs based HEMTs [4].

A semiclassical ensemble MC simulator self-consistently coupled with a twodimensional Poisson solver has been used for device simulations $[8,9]$. The simulator is properly adapted to work with narrow band-gap channel HEMTs [10]. As seen later, impact ionization becomes crucial for $V_{d s}>0.3 \mathrm{~V}$. We will focus on lower voltages (where the dynamic and noise performances are expected to be optimal). Hence impact ionization has not been considered due to the complications that introduces in the 
calculation of the small signal equivalent circuit [11]. We study impact ionization processes and the consequent kink effect in a separate work dealing only with the static behaviour of the transistors [12].

The simulated $225 \mathrm{~nm}$ gate Sb-HEMTs is shown in figure 1. In order to decrease the number of simulated particles and reduce the simulation time, the ohmic source-recess region has been shortened from the experimental value of around $1.0 \mu \mathrm{m}$ to $300 \mathrm{~nm}$. The omitted part can be introduced later as a contribution to the external source resistance [8]. To reproduce the surface charges that appear in the semiconductor-air

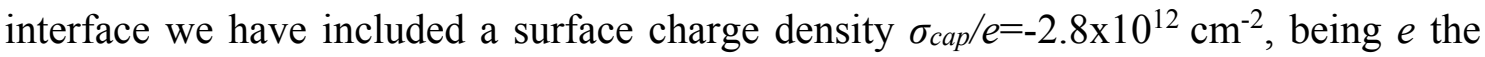
electron charge (value that reproduces the experimental sheet electron density in the channel, $\left.n_{s}=2.1 \times 10^{12} \mathrm{~cm}^{-2}\right)^{7}$. The negative surface charge at the recess floor, $\sigma_{\text {recess, }}$ may be different from the one in the top of the cap layer [8], since it depends on the etching process and the material is different. Moreover, the value assigned to $\sigma_{\text {recess }}$ also accounts for the possible presence of charges within the native oxide. Therefore, this parameter will be taken as adjustable, within reasonable values.

AlSb oxidation is a complex process [13], being difficult to know which is the composition of the oxide and its thickness. The static and dynamic behaviour of the devices has been simulated, analyzing the influence of the dielectric constant and oxide thickness. We have observed that increasing the dielectric constant is equivalent to decreasing the oxide thickness, obtaining a reasonable agreement with experimental data considering a $2 \mathrm{~nm}$ thick oxide with a dielectric constant of 2.2 (corresponding to Al hydroxide).

Three different cases have been simulated in order to study the intrinsic influence of the native oxide and the surface charges at the bottom of the recess (figure 1):

- Device without native oxide under the gate and with a surface charge on the 


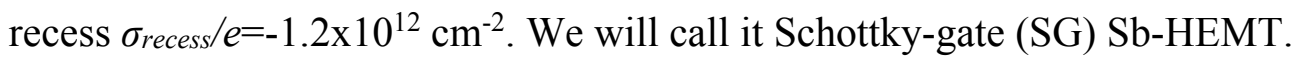

- Device with the same $\sigma_{\text {recess }}$ as the $\mathrm{SG}$, but with a $2 \mathrm{~nm}$ thick oxide with dielectric constant 2.2 under the gate. We will call it isolated-gate (IG) SbHEMT.

- Device identical to IG but with a higher negative surface charge at the bottom of the recess $\sigma_{\text {recess }} / e=-2.8 \times 10^{12} \mathrm{~cm}^{-2}(\mathrm{IG} 2)$.

\section{Results}

\section{a) Static characteristics:}

In order to compare the intrinsic $\mathrm{MC}$ results with the extrinsic experimental ones two considerations must be done. First, we have to introduce the effect of the parasitic elements which are not present in our simulations. Consequently source and drain contact resistances $R_{s}$ and $R_{d}$ have been included for a proper fitting of the IG case. The best fit was found with $R_{s}=0.13 \Omega \cdot \mathrm{mm}$ and $R_{d}=0.38 \Omega \cdot \mathrm{mm}$, in good agreement with the experimental values of the contact access resistances. The second post-processing step to be done with the intrinsic MC results is a $V_{g s}$ shift $\left(\Delta V_{g s}\right)$ associated with the gate properties. In the case of the SG Sb-HEMTs this is due to the metal-semiconductor Schottky barrier height, which is not considered in the simulations. This should introduce a positive shift of $V_{g s}$ with respect to the electrical bias applied in the MC simulations. However, in this case the $\Delta V_{g s}$ needed to correctly reproduce the experimental threshold voltage has a negative value $(-0.37 \mathrm{~V})$. This fact confirms the presence of the native oxide in the IG Sb-HEMT. This is also the reason why the reference case of the $\mathrm{MC}$ simulations used in the comparison with the experimental results is the IG-HEMT. In the SG case, the $V_{g s}$ shift does not have a physical meaning, 
but it has been kept in order to perform an understandable comparison of the different simulated devices. In the cases IG and IG2 the $\Delta V_{g s}$ introduced is attributed to the effect of i) the metal-semiconductor workfunction difference, ii) the surface charges at the semiconductor-oxide interface and iii) the volume charges within the oxide, whose global value is not known. The best fit for IG and IG2 is achieved with a $V_{g s}$ shift of $0.03 \mathrm{~V}$ and $-0.27 \mathrm{~V}$, respectively. The different $\Delta V_{g s}$ just compensates the effect of the higher $\sigma_{\text {recess }}$ in IG2 with respect to IG, which results that (in this range of values) $\sigma_{\text {recess }}$ has almost no influence on the DC and the dynamic behaviour of the devices, so that we have some flexibility for choosing its value in the MC simulations.

In figure 2, it is shown a good agreement between MC simulations of Sb-HEMTs and experimental results for low $V_{d s}$ (up to $0.2-0.3 \mathrm{~V}$ ), when impact ionization mechanisms are not frequent, especially in the case of IG and IG2. For higher $V d s$ a very high output conductance resulting from impact ionization appears in the experimental output I-V curves, figure 2(a), with a subsequent threshold voltage shift in the transfer characteristics, figure 2(b), not reproduced by the simulations. Figure 2 shows that IG and IG2 nearly provide the same DC results, since the variation of the value of $\sigma_{\text {recess }}$ only results in a different threshold voltage (that is compensated by the difference in the $\Delta V_{g s}$ used to adjust the $\mathrm{MC}$ characteristics) without much affecting the final shape of the I-V curves. It can also be observed that the absence of oxide under the gate (SG case) somewhat increases the drain current as a consequence of a better charge control by the gate. This is the penalty that the IG design pays for the reduction of the gate leakage current. Note that the gate current is not shown in the simulations since it cannot be calculated with our semiclassical MC model.

\section{b) Dynamic results}

The simulated and experimental results for the intrinsic small signal equivalent 
circuit parameters of the SG and IG HEMTs are compared in figure 3 for $V_{d s}=0.2 \mathrm{~V}$, where impact ionization is negligible and the optimum noise conditions are experimentally reached [6]. The MC results have been obtained from the admittance parameters extracted by Fourier analysis of the response of the transistor currents to voltage steps applied at the gate and drain contacts [14]. In order to perform a correct comparison between the intrinsic experimental and simulated equivalent circuits we have to consider that even when deembedding the extrinsic parameters from the experimental results, there are still some effects the MC is not accounting for, such as fringing and cross talk capacitances in the layout. Indeed, three extrinsic capacitances, $C_{g s}^{e x t r}, C_{g d}^{e x t r}$ and $C_{d s}^{e x t r}$, have been added to the MC results to reproduce the effects associated with the topology of the devices (see Ref. 14 for a more detailed explanation). The values of $C^{\text {extr }}$ have been obtained by fitting the experimental data at zero current $\left(C_{d s}^{\text {extr }}=120 \mathrm{fF} / \mathrm{mm}, C_{g d}^{\text {extr }}=120 \mathrm{fF} / \mathrm{mm}, C_{g s}^{\text {extr }}=10 \mathrm{fF} / \mathrm{mm}\right)$, where it remains only the effect of the geometric capacitances, which are independent of the applied bias.

The intrinsic equivalent circuit combined with the $C^{\text {extr }}$ is compared in figure 3 with the intrinsic experimental small-signal equivalent circuit. The simulated IG and IG2 cases are very similar, thus confirming that the surface and volume charges below the gate produce only a threshold voltage shift without influencing the dynamic behaviour of the devices. These two simulated cases (IG and IG2) provide a better agreement with the experimental results for $C_{g s}$ than the SG one, while for $C_{g d}$ the gate oxide does not have a strong influence [figure 3(a)-(b)]. The increased value of both $C_{g s}$ and $C_{g d}$ in the SG case is consistent with the increased gate-channel capacitance in the absence of the gate oxide.

The existence of a frequency dependence in the experimental results of $C_{d s}$ and $g_{d}$ causes the considerable disagreement between the experimental and MC results 
observed in figure 3(c) and (d). We attribute this frequency dispersion to the existence of holes in the buffer whose response time is quite slow. This effect has already been observed in InGaAs HEMTs [11,15], even under biases where the kink effect is still not apparent in the I-V curves. When holes are accumulated, $g_{d}$ shows a frequency dispersion associated to their accumulation time (related to impact ionization processes and the time which holes take to move from the region where they are generated to the accumulation zone).

The presence of the gate oxide reduces $g_{m}$ in the IG with respect to the SG device [figure 3(e)], as expected from the inferior charge control shown figure 2. Consequently, also a decrease is observed in the intrinsic cut-off frequency $f_{c}=g_{m} / 2 \pi\left(C_{g s}+C_{g d}\right)$ for the IG cases. $f_{c}$ is plotted in figure 3(f). The lower $g_{m}$ is partially compensated by a decrease of $C_{g s}$.

For the IG cases in figure 3(e), the maximum intrinsic $g_{m}$ and the drain current are lower in the $\mathrm{MC}$ simulations than for the experimental curve This disagreement was unforeseen, mainly because a much better agreement exists when the extrinsic $g_{m}$ is extracted from the static I-V curves (figure 2, IG case). Indeed, the values for the contact resistances to be added to MC simulations were chosen in order to obtain the best fit for the extrinsic I-V characteristics. This inconsistency can be explained by the discrepancy in the drain conductance [figure $3(\mathrm{~d})$ ], since $g_{m}^{\text {extr }}$ and $g_{m}^{\text {int } r}$ are coupled by the relation

$$
g_{m}^{\text {extr }}=\frac{g_{m}^{\text {int } r}}{\left(1+g_{m}^{\text {int } r} R_{S}\right)\left[1+g_{d}^{\text {int } r}\left(R_{S}+R_{d}\right)\right]}
$$

In fact, the extrinsic $g_{m}$ obtained using Eq. (1), plotted in figure 4, show that the maximum values of the IG MC and experimental extrinsic $g_{m}$ are similar, even if the shape of the curve is not really the same (the peak is found at lower currents in the 
simulations). In order to better reproduce the experimental results we have considered another possible source of discrepancies between the experimental and simulated results, which is the variation of the value of the sheet electron density in the channel. The results of Hall measurements used in MC simulations were obtained in the epitaxial structure before device processing. During device processing, the value of $n_{s}$ may be affected. A higher sheet electron density $\left(n_{s}=3.3 \times 10^{12} \mathrm{~cm}^{-2}\right)$ has been achieved in our

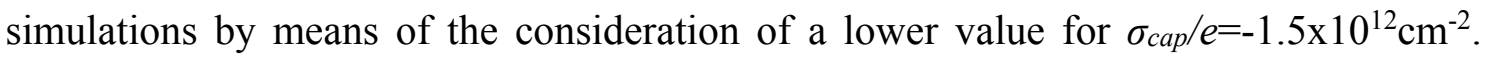
However, this just affects the source and drain access regions and not the active part of the device, thus decreasing the source and drain access resistances due to the higher $n_{s}$ on those regions (the electron density under the recess and the gate practically do not change). However, we have to note here that the values for the extrinsic resistances added to all the intrinsic MC simulations have been kept constant, so that the total contact resistance decrease would be even higher.

Figure 4, shows that the peak in $g_{m}$ is shifted to higher $I_{d}$ when $n_{s}$ is higher, thus providing a much better agreement with the experimental values. The same trend is followed by the intrinsic $g_{m}$, as can be observed in figure 5, where the comparison between the SG and IG MC cases with the two values of $n_{s}\left(2.1 \times 10^{12} \mathrm{~cm}^{-2}\right.$ and $3.3 \times 10^{12} \mathrm{~cm}^{-2}$ ) is shown. If we focus on the IG-MC simulation, the decrease of the source and drain access resistances due to the higher $n_{s}$ also leads to a slight reduction in the values of $C_{g s}$, while those of $C_{g d}$ remain similar, as show figure 6 . The slight decrease of Cgs (while Cgd remains practically constant) is the result of a complex bidimensional electrostatic effect, resulting from the influence of sigma_cap at the lateral parts of the active region of the device (mainly at the source side). This is the result of a bi-dimensional electrostatic effect, resulting from the influence of $\sigma_{c a p}$ in the lateral parts of the active region of the device (mainly at the source side). 
In order to discern the importance of each of the studied parameters on the extrinsic high frequency performance of the devices, figure 7 shows the extrinsic current gain cut-off frequency, $f_{t}$, estimated as [16]:

$$
f_{t}=\frac{g_{m}^{\text {int } r} / 2 \pi}{\left(C_{g s}+C_{g d}\right)\left[1+g_{d}^{\text {int } r}\left(R_{S}+R_{d}\right)\right]+C_{g d} g_{m}^{\text {int } r}\left(R_{s}+R_{d}\right)} .
$$

Not many differences are found between the $f_{t}$ values obtained for the IG and SG cases, thus showing that a thin gate oxide helps to reduce the gate leakage current without much degrading the high frequency performance of the HEMTs. On the other hand, an increased value of $n_{s}$ makes the peak $f_{t}$ to be shifted to higher $I_{d}$ (and slightly increase its value) in a similar way as happened with $g_{m}$, providing a reasonable agreement with the experimental results. The lower experimental values of $f_{t}$ with respect to the $\mathrm{MC}$ ones are mainly due to the discrepancy in the $g_{d}$ values.

\section{CONCLUSIONS}

The static and dynamic behaviour of InAs/AlSb isolated-gate Sb-HEMTs has been studied by MC simulations and compared with experimental results, with which a reasonable agreement has been obtained for $V_{d s}<0.3 \mathrm{~V}$, when impact ionization processes are not frequent. Discrepancies arise for $g_{d}$ and $C_{d s}$ at high drain current due to the frequency dispersion of the experimental measurements, that we attribute to the presence of holes in the buffer originated by impact ionization in the InAs channel. This, added to the relation between the intrinsic and extrinsic $g_{m}$, where $g_{d}$ is involved, makes the simulated intrinsic dynamic $g_{m}$ values depart from the experimental ones for high $I_{d}$, while the static extrinsic $g_{m}$ is well reproduced.

We have shown that the native oxide under the gate used to reduce the gate leakage current leads to a decrease in $g_{m}$, due to the expected poorer channel charge control by 
the gate, and also in $C_{g s}$ and $f_{c}$, but it does not degrade significantly the extrinsic high frequency performance of the IG HEMTs (estimated through $f_{t}$ ).

When different values for the surface charges at the bottom of the recess of the IG HEMTs are used in the simulation, only a threshold voltage shift is observed in both static and dynamic characteristics, meaning that there is some flexibility for the choice of this MC parameter. When increasing the $n_{s}$ value in the MC simulations, a better agreement with the experimental shape of $g_{m}$ and $f_{t}$ is obtained (the maximum shifts towards higher $\mathrm{I}_{\mathrm{d}}$ ), thus indicating the possibility that the sheet electron density in the channel of the Sb-HEMTs were modified during the device processing.

\section{ACKNOWLEDGMENTS}

This work has been partially supported by the European Commission through the ROOTHz Project ICT-2009-243845 and the MC2ACCESS Project, by the Dirección General de Investigación (MEC) and FEDER through Project TEC2007-61259/MIC and by the Consejería de Educación, Junta de Castilla y León through Projects SA019A08 and GR270. 


\section{REFERENCES}

[1] Kroemer H 2004 The $6.1 \AA$ family (InAs, GaSb, AlSb) and its heterostructures: a selective review Physica E 20196

[2] Boos J B, Kruppa W, Bennett B R, Park D, Kirchoefer S W, Bass R and Dietrich H B $1998 \mathrm{AlSb} /$ InAs HEMTs for low voltage, high-speed applications IEEE Trans. Electron Devices 451869

[3] Bolognesi C R, Dvorak M W and Chow D H 1999 Impact ionization suppression by quantum confinement: effects on the DC and microwave performance of narrowGap channel InAs/AlSb HFET’s IEEE Trans. Electron Devices 46826

[4] Bennett B R., Magno R., Boos J B, Kruppa W and Ancona M G 2005 Antimonidebased compound semiconductors for electronic devices: A review. Solid-State Electron. 491875

[5] Kruppa W, Boos B, Bennett B R, Papanicolaou N A, Park D and Bass R 2007 Lowfrequency noise in A1Sb/InAs and related HEMNTs. IEEE Trans. Electron Devices 541193

[6] Malmkvist M, Lefebvre E, Borg M, Desplanque L, Wallart X, Dambrine G, Bollaert S and Grahn J 2008 Electrical characterization and small-signal modelling of InAs/AlSb HEMTs for low-noise and high-frequency applications IEEE Trans. Microw. Theory Technol. 562685

[7] Lefebvre E, Malmkvist M, Borg M, Desplanque L, Wallar X, Dambrine G, Bollaert S and Grahn J 2009 IEEE Trans. Electron Devices 561904

[8] Mateos J, González T, Pardo D, Hoel V, Happy H and Cappy A 2000 Improved Monte Carlo algorithm for the Simulation of $\delta$-doped AlInAs/GaInAs HEMT's IEEE Trans. Electron Devives $\mathbf{4 7} 250$ 
[9] Mateos J, González T, Pardo D, Hoel V and Cappy A 1999 Effect of the T-gate on the performance of recessed HEMTs. A Monte Carlo analysis Semicond. Sci Technol. 14864

[10] Rodilla H, González T, Pardo D and Mateos J 2009 High-mobility heterostructures based on InAs and InSb: A Monte Carlo study J. Appl. Phys. 105113705

[11] Vasallo B G, Mateos J, Pardo D and González T 2005 Influence of the kink effect on the dynamic performance of short-channel InAlAs/InGaAs high electron mobility transistors Semicond. Sci. Technol. 20956

[12] Vasallo B G, Rodilla H, González T, Moschetti G, Grahn J and Mateos J submitted

[13] Shibata T, Nakata J, Nanishi Y and Fujimoto M 1994 A Rutherford backscattering spectroscopic study of the Aluminium Antimonide oxidation process in air Jpn. J. Appl. Phys. 331767

[14] Mateos J, González T, Pardo D, Höel V and Cappy A 2000 Monte Carlo Simulator for the design optimization of low-noise HEMTs IEEE Trans. Electron Devices $\mathbf{4 7}$ 1050

[15] Vasallo B G, Mateos J, Pardo D and González T 2004 Kink-effect related noise in short-channel InAlAs/InGaAs high electron mobility transistors J. Appl. Phys. 95 8271

[16] Tasker P J and Hughes B 1989 Importance of source and drain resistance to the maximum $f_{T}$ of millimeter-wave MODFETsIEEE Electron Device Lett., 10291 


\section{FIGURE CAPTIONS}

Figure 1. Scheme of the simulated devices.

Figure 2. Comparison of the experimental (black solid lines) and MC (symbols, dashed colored lines) results: green triangles for $\mathrm{SG}$, red circles for IG, and blue diamonds for IG2 HEMTs. (a) $I_{d}-V_{d s}$ from $V_{g s}=-1.15 \mathrm{~V}$ to $-0.45 \mathrm{~V}$, step $0.1 \mathrm{~V}$ and (b) $I_{d}-V_{g s}$ from $V_{d s}=0.1 \mathrm{~V}$ to $0.6 \mathrm{~V}$, step $0.1 \mathrm{~V}$.

Figure 3. Comparison between the values of the (a) gate-source, $C_{g s}$, (b), gate-drain, $C_{g d}$, and (c) drainsource, $C_{d s}$, capacitances, (d) drain conductance, $g_{d}$, (e) transconductance, $g_{m}$, and (f) intrinsic cutoff frequency $f_{c}=g_{m} / 2 \pi\left(C_{g s}+C_{g d}\right)$ measured in the fabricated HEMT (black line) and obtained by MC simulations for the SG (green triangles), IG (red circles), and IG2 (blue diamonds) cases as a function of $I_{d}$ for $V_{d s}=0.2 \mathrm{~V}$.

Figure 4. Extrinsic $g_{m}$ calculated through eq. (1) for $V_{d s}=0.2 \mathrm{~V}$ for the fabricated HEMT (black solid line) as a function of $I_{d}$ compared with the IG MC results for two different $\sigma_{c a p}$ values leading to $n_{s}=2.1 \times 10^{12} \mathrm{~cm}^{-2}$ (red circles, dashed red line) and $n_{s}=3.3 \times 10^{12} \mathrm{~cm}^{-2}$ (white circles, solid red line).

Figure 5. Intrinsic $g_{m}$ for $V_{d s}=0.2 \mathrm{~V}$ for the fabricated HEMT (black solid line) as a function of $I_{d}$ compared with the MC intrinsic results, SG (triangles) and IG (circles), for the two values of $n_{s}$ considered, $\left(n_{s}=2.1 \times 10^{12} \mathrm{~cm}^{-2}\right.$ colored symbols, dashed lines, $n_{s}=3.3 \times 10^{12} \mathrm{~cm}^{-2}$ white symbols, solid lines).

Figure 6. Comparison between the intrinsic values of the (a) gate-source, $C_{g s}$, and (b), gate-drain, $C_{g d}$, capacitances obtained by MC simulations of the IG device for the two values of $n_{s}$ considered: $n_{s}=2.1 \times 10^{12} \mathrm{~cm}^{-2}$ (colored red circles, dashed lines), and $n_{s}=3.3 \times 10^{12} \mathrm{~cm}^{-2}$ (white circles, red solid lines) as a function of $I_{d}$ for $V_{d s}=0.2 \mathrm{~V}$. 
Figure 7. $\mathrm{f}_{\mathrm{t}}$ estimated through eq. (2) as a function of $I_{d}$ for $V_{d s}=0.2 \mathrm{~V}$ in the fabricated HEMT, compared with the SG and IG MC results with two different values of $n_{s}$. 


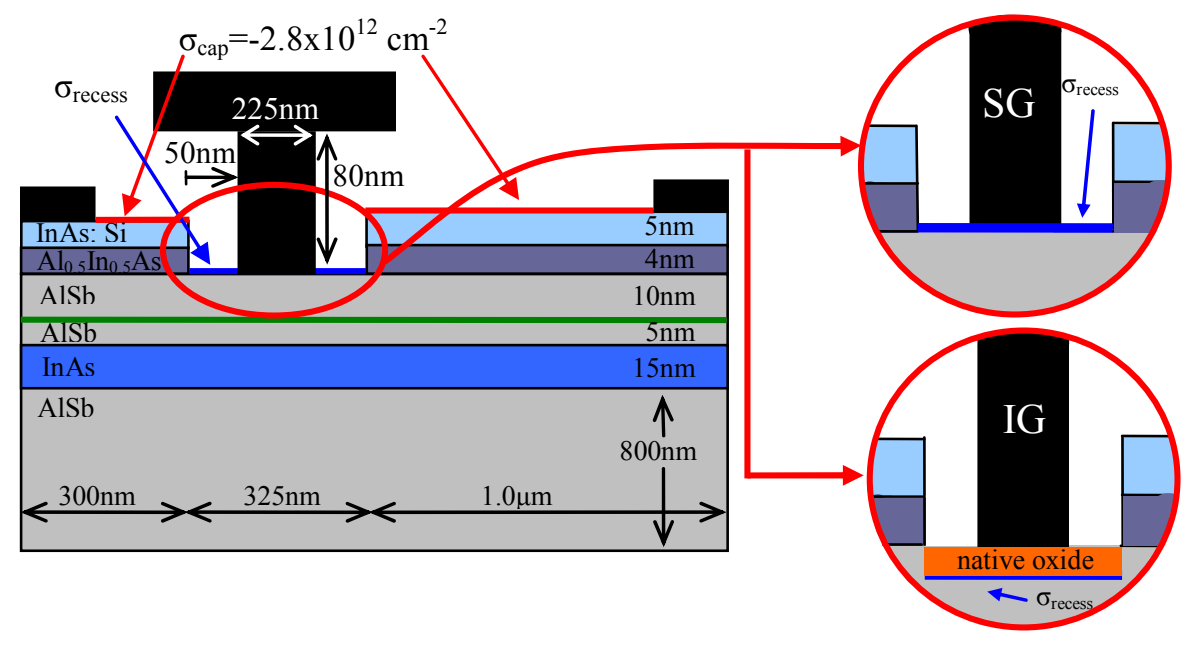

Figifure 1. Scheme of the simulated devices. 

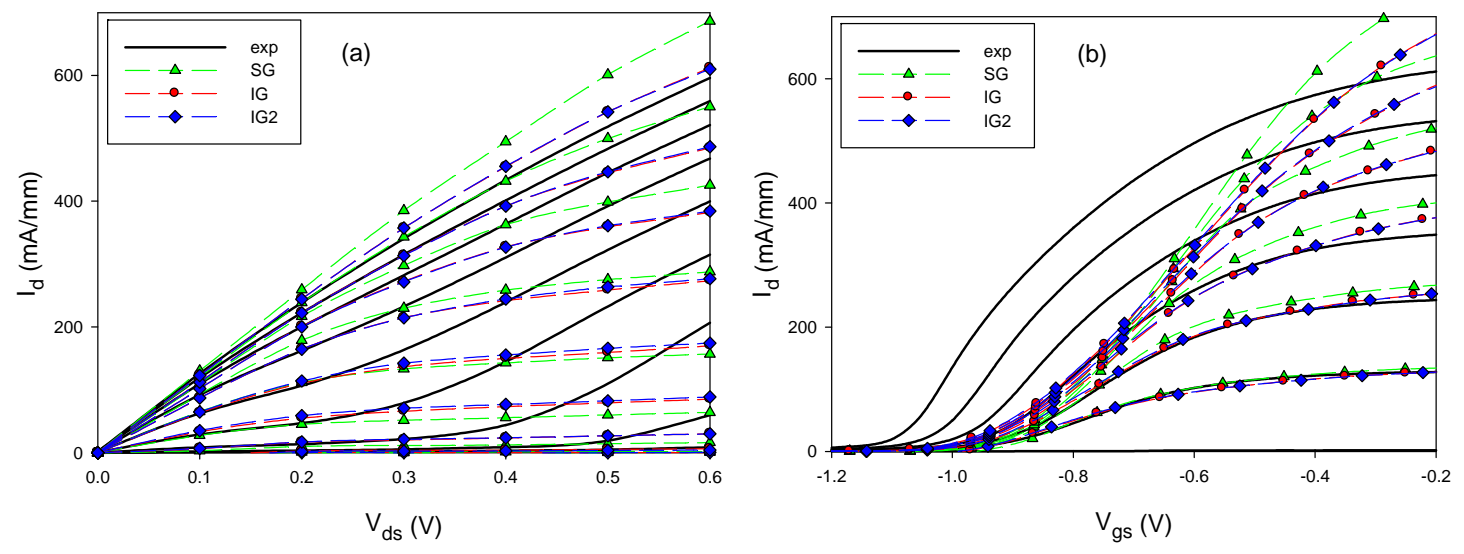

Figure 2. Comparison of the experimental (black solid lines) and MC (symbols, dashed colored lines) results: green triangles for SG, red circles for IG, and blue diamonds for IG2 HEMTs. (a) $I_{d}-V_{d s}$ from $V_{g s}=-1.15 \mathrm{~V}$ to $-0.45 \mathrm{~V}$, step $0.1 \mathrm{~V}$ and (b) $I_{d^{-}} V_{g s}$ from $V_{d s}=0.1 \mathrm{~V}$ to $0.6 \mathrm{~V}$, step $0.1 \mathrm{~V}$. 

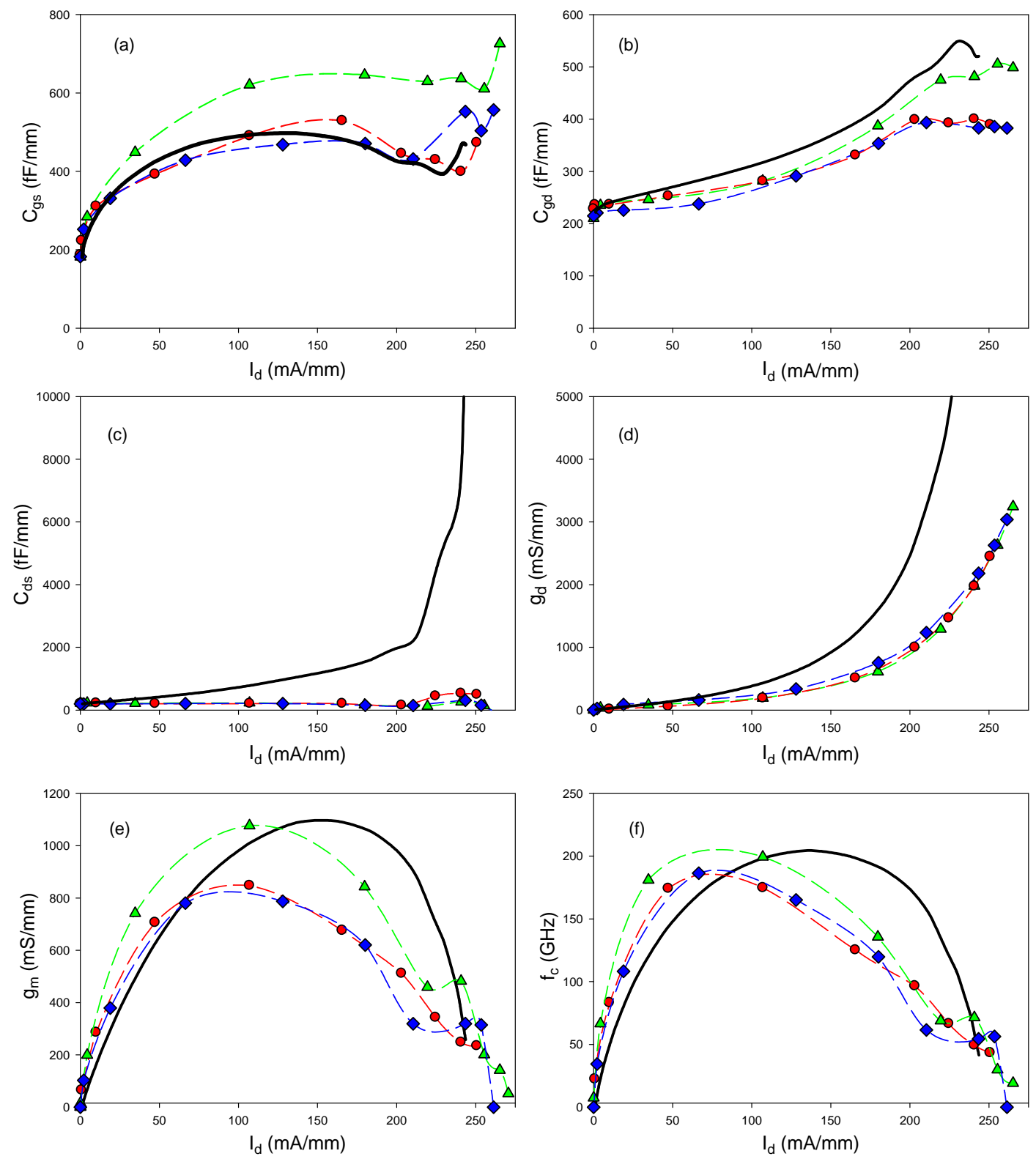

Figure 3. Comparison between the values of the (a) gate-source, $C_{g s}$, (b), gate-drain, $C_{g d}$, and (c) drainsource, $C_{d s}$, capacitances, (d) drain conductance, $g_{d}$, (e) transconductance, $g_{m}$, and (f) intrinsic cutoff frequency $f_{c}=g_{m} / 2 \pi\left(C_{g s}+C_{g d}\right)$ measured in the fabricated HEMT (black line) and obtained by MC simulations for the SG (green triangles), IG (red circles), and IG2 (blue diamonds) cases as a function of $I_{d}$ for $V_{d s}=0.2 \mathrm{~V}$. 


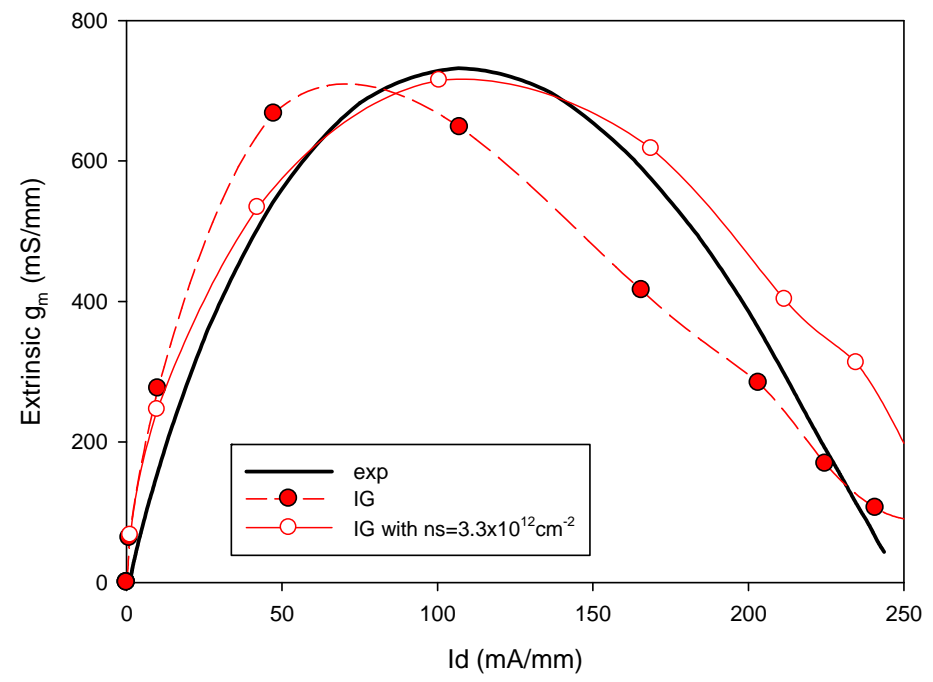

Figure 4. Extrinsic $g_{m}$ calculated through eq. (1) for $V_{d s}=0.2 \mathrm{~V}$ for the fabricated HEMT (black solid line) as a function of $I_{d}$ compared with the IG MC results for two different $\sigma_{c a p}$ values leading to $n_{s}=2.1 \times 10^{12} \mathrm{~cm}^{-2}$ (red circles, dashed red line) and $n_{s}=3.3 \times 10^{12} \mathrm{~cm}^{-2}$ (white circles, solid red line). 


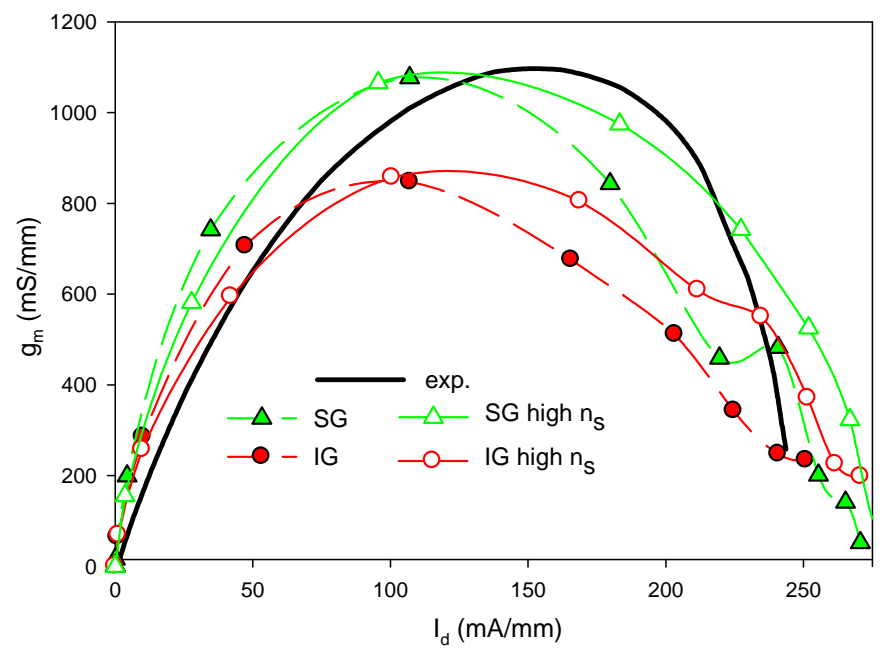

Figure 5. Intrinsic $g_{m}$ for $V_{d s}=0.2 \mathrm{~V}$ for the fabricated HEMT (black solid line) as a function of $I_{d}$ compared with the MC intrinsic results, SG (triangles) and IG (circles), for the two values of $n_{s}$ considered, $\left(n_{s}=2.1 \times 10^{12} \mathrm{~cm}^{-2}\right.$ colored symbols, dashed lines, $n_{s}=3.3 \times 10^{12} \mathrm{~cm}^{-2}$ white symbols, solid lines $)$. 


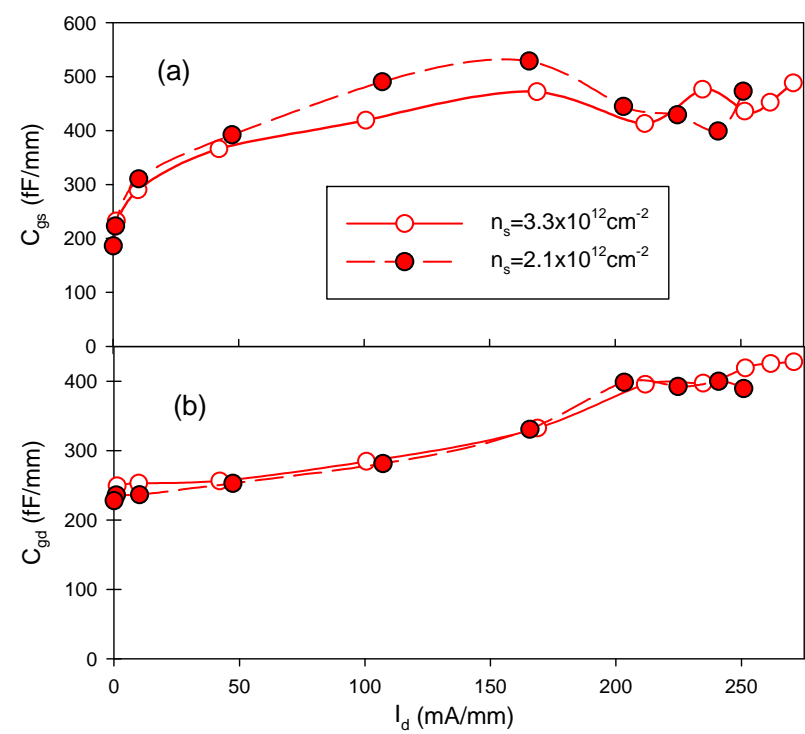

Figure 6. Comparison between the intrinsic values of the (a) gate-source, $C_{g s}$, and (b), gate-drain, $C_{g d}$, capacitances obtained by MC simulations of the IG device for the two values of $n_{s}$ considered: $n_{s}=2.1 \times 10^{12} \mathrm{~cm}^{-2}$ (colored red circles, dashed lines), and $n_{s}=3.3 \times 10^{12} \mathrm{~cm}^{-2}$ (white circles, red solid lines) as a function of $I_{d}$ for $V_{d s}=0.2 \mathrm{~V}$. 


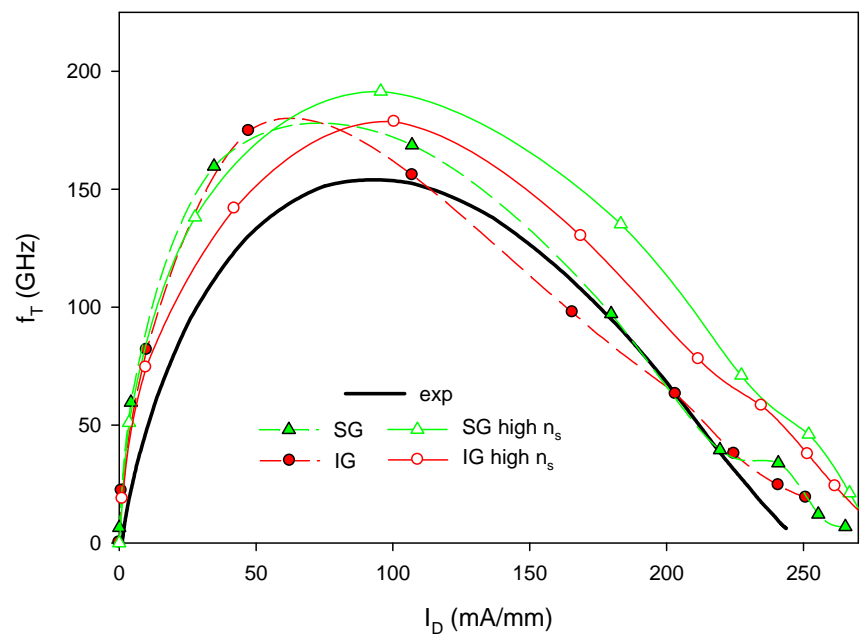

Figure 7. $\mathrm{f}_{\mathrm{t}}$ estimated through eq. (2) as a function of $I_{d}$ for $V_{d s}=0.2 \mathrm{~V}$ in the fabricated HEMT, compared with the SG and IG MC results with two different values of $n_{s}$. 